\title{
SOME ESTIMATES FOR HARMONIC MEASURES. II
}

\author{
JAMES A. JENKINS
}

(Communicated by Clifford J. Earle, Jr.)

\begin{abstract}
FitzGerald, Rodin, and Warschawski proved that, for a continuum of given diameter in the closed unit disc, the harmonic measure at the center is minimized when it is an arc on the circumference. A very simple proof of this result is given, using the method of the extremal metric.
\end{abstract}

1

In [1], FitzGerald, Rodin, and Warschawski gave the solution for two extremal problems for the harmonic measure of a continuum lying in the unit disc. These results may be stated as follows, using the following notations: $F$ is the closed unit disc $|z| \leq 1, E$ the open unit disc, $C$ a continuum in $F$ not containing the origin, $G$ the component of $E-C$ containing the origin, $\alpha$ the border entity of $G$ determined by $C$, and $\omega(0, \alpha, G)$ the harmonic measure of $\alpha$ at 0 with respect to $G$.

I. If $C$ has diameter $\delta, \omega(0, \alpha, G) \geq \frac{1}{2 \pi} \theta$ where $\theta$ is the angular measure of an arc on $|z|=1$ of diameter $\delta$, with equality precisely in that case.

II. If $C$ subtends an angle of measure $\theta$ equal at most to $\pi$ at $0, \omega(0, \alpha, G) \geq$ $\frac{1}{2 \pi} \theta$ with equality if and only if $C$ is an arc of angular measure $\theta$ on $|z|=1$.

In [2], the present author pointed out that by using triad modules a very simple proof of II can be given by the method of the extremal metric. Now we will observe that I is an immediate consequence of the extremal property of the Mori extremal domain.

2

The extremal property of the Mori extremal domain [3] can be stated as follows:

III. Let $C^{*}$ be a continuum not containing the origin or the point at infinity which has two points in $|z| \leq 1$ of distance $\geq \delta>0$. Let $\Gamma^{*}$ be the homotopy

Received by the editors October 3, 1989.

1980 Mathematics Subject Classification (1985 Revision). Primary 31A15, 30C85. 
class of rectifiable Jordan curves separating $C^{*}$ from 0 and $\infty$. Then the module $m\left(\Gamma^{*}\right)$ of $\Gamma^{*}$ is maximal precisely when $C^{*}$ is an arc of diameter $\delta$ on $|z|=1$.

To derive I from III, we remark first, as in [2], that we may assume $C$ meets $|z|=1$. Then $I$ is equivalent to maximizing the triad module $m(0, \beta, G)$ where $\beta$ is the open boundary arc of $G$ on $|z|=1$. Let $\widetilde{C}$ be the reflection of $C$ in $|z|=1, C^{*}=C \cup \widetilde{C}$. Evidently $m(0, \beta, G)=2 m\left(\Gamma^{*}\right)$. Now I follows at once from III.

\section{BIBLIOGRAPHY}

1. C. FitzGerald, B. Rodin, and S. Warschawski, Estimates of the harmonic measure of a continuum in the unit disc, Trans. Amer. Math. Soc. 287 (1985), 681-685.

2. James A. Jenkins, Some estimates for harmonic measures, in Complex Analysis I (Proc. of the Special Year, University of Maryland, College Park, 1985-86), Lecture Notes in Math., vol. 1275, Springer-Verlag, 1987, pp. 210-214.

3. A. Mori, On an absolute constant in the theory of quasiconformal mappings, J. Math. Soc. Japan 8 (1956), 156-166.

Department of Mathematics, Washington University, St. Louis, Missouri 63130 